\title{
Cobertura de la Citología de Cuello Uterino y Factores Relacionados en Colombia, 2005
}

Pap test coverage and related factors in Colombia, 2005

Marion Piñeros, Ricardo Cendales, Raúl Murillo, Carolina Wiesner y Sandra Tovar

Instituto Nacional de Cancerología, Subdirección de Investigaciones, Bogotá, Colombia mpineros@cancer.gov.co

Recibido 20 Octubre 2006/Enviado para Modificación 6 Junio 2007/Aceptado 1 Agosto 2007

RESUMEN

Objetivo Describir la cobertura de la citología en los últimos tres años y los principales factores relacionados, en la población de mujeres colombianas entre 25 y 69 años de edad.

Materiales y métodos Se analizó la información de la Encuesta Nacional de Demografía y Salud 2005. Se calculó la variable "práctica de la citología en los últimos tres años". La descripción de la cobertura y factores asociados se hizo a través de porcentajes simples y se realizó un análisis multivariado utilizando un modelo de regresión logística incondicional.

Resultados La cobertura de citología reciente en mujeres entre 25 y 69 años de edad para Colombia fue de 76,5\%. Las condiciones asociadas con la ausencia de toma reciente fueron ausencia de afiliación, afiliación al régimen subsidiado, no haber tenido hijos vivos ni una consulta de salud en el último año; las condiciones asociadas con la toma reciente fueron el nivel de riqueza, tener entre 35 y 44 años, tener un nivel educativo universitario o superior y estar en embarazo.

Conclusión Los resultados indican la necesidad de esfuerzos por mejorar las coberturas en la población más pobre, no afiliada y afiliada al régimen subsidiado; la alta mortalidad a pesar de la buena cobertura general sugiere la necesidad de esfuerzos en la oportunidad del diagnóstico definitivo y el manejo de lesiones.

Palabras Clave: Cuello uterino, citología, prevención de cáncer de cuello uterino, neoplasias (fuente: DeCS, BIREME)

\section{ABSTRACT}

Objective Describing the use of the Papanicolau (Pap) test and the main factors related to using screening amongst Colombian women aged 25 to 69 years.

Methods Information was taken from the 2005 National Health and Demography Survey. The variable "pap test during the last three years" was calculated; coverage and related factors were described using simple percentages and multivariate analysis using conditional logistic regression.

Results Recent Pap test coverage in Colombian women aged 25 to 69 years was 
$76.5 \%$. Factors associated with the absence of recent exam included non-affiliation to the health system, affiliation to the susbsidiary regimen, having no live-born children and no recent medical consultation. Factors associated with recent coverage were income level, being aged 35 to 44, a higher educational level and being pregnant. Conclusions Results indicated the need for efforts at increasing coverage amongst the poorer population as well as amongst women from the subsidiary regimen and those having no affiliation. Persistence of high mortality in spite of acceptable coverage suggested the need for more efforts regarding definitive diagnosis and opportune treatment.

Key Words: Cervix uteri, neoplasm, surveys, prevention (source: MeSH, NLM)

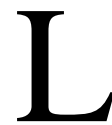

a efectividad de la citología cervico-uterina como prueba de tamización ha sido claramente demostrada en diferentes países observándose importantes reducciones de la mortalidad por cáncer de cuello uterino (1-3). Sin embargo en muchos países en vías de desarrollo que han implementado programas de detección temprana basados en la citología, la reducción no ha sido la esperada (4).

A nivel mundial el cáncer de cuello uterino es el segundo tipo de cáncer más común en la mujer y del total de muertes por este cáncer en el año 2002, el $85 \%$ ocurrieron en países en vías de desarrollo (5). En Colombia, para el año 2002 se estimaron 6815 casos nuevos de cáncer de cuello uterino (5) y se registraron 2045 muertes por esta causa con una tasa de mortalidad ajustada por edad de 10,8 por 100000 mujeres, lo que la sitúa como la primera causa de muerte por cáncer y además la primera causa relacionada con la salud sexual y reproductiva (6).

Adicionalmente si se ajustan las muertes por subregistro la tasa estimada de mortalidad podría alcanzar los 18,1 por 100000 mujeres (7). El análisis del comportamiento de la enfermedad revela que la tendencia de la mortalidad en Colombia de los últimos quince años, aunque muestra un leve descenso, se ha mantenido sin mayor variación (8) a pesar de que en 1991 se creó un programa nacional de detección precoz (9) y que dentro del actual Sistema General de Seguridad Social las actividades para el control de esta patología han sido una prioridad en salud pública $(10,11)$. Las normas vigentes estipulan la realización de la citología entre los 25 a 69 años de edad, en un esquema que incluye una citología negativa inicial, una citología negativa al año y luego controles cada tres años si la citología sigue siendo negativa; este esquema se conoce como esquema 1-1-3 y será referido de esta forma en desarrollo del artículo (10). 
En relación con la cobertura esperada a nivel poblacional, la norma definió metas programáticas de cumplimiento de $80 \%$ aplicables a mujeres afiliadas al régimen contributivo y subsidiado (12); sin embargo estas metas no discriminan el comportamiento de las mujeres en relación con el esquema y por ende la información resultante no puede interpretarse como cobertura.

De acuerdo con el esquema propuesto y con las recomendaciones a nivel mundial (13) es necesario tener en cuenta el periodo de tiempo al que hace referencia la cobertura que para el caso es la realización de una citología en los últimos tres años, y se define éste como indicador de riesgo para cáncer de cuello uterino (14).

El Estudio Nacional de Factores de Riesgo para Enfermedades Crónicas (15)-ENFRECII- arrojó información de cobertura de citología cervicouterina en los últimos tres años con representatividad a nivel regional.

Con base en la Encuesta Nacional de Demografía y Salud 2005 -ENDS 2005- se realizó un análisis de la cobertura de la citología en los últimos tres años y los principales factores relacionados (16).

El objetivo de este artículo es presentar los resultados descritos sobre la población objeto de las actividades de detección temprana (mujeres de 25 a 69 años), discriminando las coberturas por departamento, lo que constituye información no disponible previamente en el país a partir de encuestas poblacionales. El análisis de factores relacionados podría ayudar a identificar aspectos que pudieran relacionarse con el comportamiento de la mortalidad por cáncer de cuello uterino en algunos departamentos.

El presente estudio busca contribuir con una evaluación de las actividades de detección temprana de cáncer de cuello uterino en el país.

\section{MATERIALES Y MÉTODOS}

El estudio fue aprobado por el Comité de Ética e Investigaciones del Instituto Nacional de Cancerología. El análisis se desarrolló en Bogotá, entre marzo y julio de 2006.

Fuentes de información

Se emplearon como fuentes de información las bases de datos de la ENDS 2005; esta encuesta de cobertura nacional, permite hacer estimaciones precisas 
según área urbana o rural y además por departamento de residencia habitual. La muestra es probabilística, por conglomerados, multietápica, estratificada y tiene como universo de estudio a la población civil, no institucional del país. Otros detalles técnicos de la encuesta pueden ser consultados en el informe original (16).

Esta fue la primera vez que se aplicó un módulo de citología en la ENDS. En consecuencia, la población objeto tradicional de la encuesta de mujeres entre 13 y 49 años, se amplió incluyendo a las mujeres entre 50 y 69 años. Esta reforma implicó modificar el muestreo, incluir las preguntas de citología en el módulo de mujeres de 13 a 49 años y diseñar un cuestionario específico para las mujeres mayores de 49 años. Este módulo se aplicó a todas las mujeres de 13 a 69 años que habían tenido relaciones sexuales vaginales y que habían oído hablar previamente sobre la citología del cuello uterino.

Para el análisis se conformó una base de datos fusionada que combinó las bases de datos de citología, hogares y personas. Se empleó como identificador único al código compuesto por el segmento, la vivienda, el hogar y el código de la persona que respondió la encuesta. Este procedimiento permitió relacionar variables de tipo socioeconómico, presentes en las bases de datos de hogares y personas, con la práctica de la citología de cuello uterino.

Para desarrollar la ENDS 2005 se seleccionaron 37211 hogares, concentrados en 3935 segmentos ubicados en 33 departamentos del país; las áreas rurales de la Orinoquía y la Amazonía fueron excluidas del universo y por lo tanto no fueron muestreadas. En las demás regiones, la muestra de segmentos se distribuyó proporcionalmente en las áreas rural y urbana.

Medición de la cobertura y factores relacionados

La práctica de la citología en los últimos tres años se calculó a partir de la fecha de la encuesta y la fecha reportada de la última citología. En aquellos casos en los que la mujer recordaba el año pero no el mes en el que se había tomado la última citología, se optó por reemplazar la información del mes faltante por el mes de junio.

La cobertura de la citología en los últimos tres años se calculó mediante la proporción de mujeres encuestadas que se hicieron la citología en los últimos tres años con respecto al total de mujeres de 25 a 69 años. 
Además de la cobertura de citología en los últimos tres años, la encuesta permitió analizar también otras variables de riesgo para cáncer de cuello uterino como la edad de la primera relación sexual, el número de hijos, el nivel educativo y el índice de riqueza. Este índice se define en términos de activos o riqueza en vez de ingresos o consumo y replica una metodología desarrollada por el Banco Mundial; otros detalles acerca del cálculo de este índice pueden ser consultadas en el informe de la ENDS 2005 (16).

\section{Métodos estadísticos}

Para todas las estimaciones obtenidas se usó el factor de expansión final, ajustado por no respuesta, sugerido por la ENDS 2005; este factor no restituye el universo de estudio sino que pondera cada elemento alrededor de la unidad, permitiendo así el cálculo no sesgado de cualquier tipo de indicador en cualquiera de los niveles de agregación regional de la muestra.

La descripción de la cobertura se hizo a través de porcentajes simples, de igual manera que la descripción de factores asociados con la toma de citología. Con el fin de controlar la confusión que pudiese existir entre el nivel educativo, la pobreza y el área de residencia con la práctica de citología, se realizó un análisis multivariado utilizando un modelo de regresión logística incondicional, en el que se incluyeron únicamente las encuestas de mujeres entre 25 y 49 años. Se verificó el supuesto de ausencia de multi colinearidad en las variables incluidas en el modelo; aquellas que resultaron colineales fueron excluidas. Para el análisis multivariado no se consideraron los factores de expansión muestral; en consecuencia los resultados de este análisis no se refieren a los datos expandidos, sino a los datos crudos. La fusión y el análisis de las bases de datos se hicieron con el software SPSS versión 11.5.

\section{RESULTADOS}

La base de datos original ponderada por el factor de expansión final descrito, tenía 41324 registros de mujeres entre 18 y 69 años; sólo se incluyeron para el análisis los registros de mujeres entre 25 y 69 años (34 674 registros). Se excluyeron 21 registros $(0,06 \%)$ en los que no se pudo establecer la edad y 6 $(0,01 \%)$ porque no se pudo establecer la condición con respecto a la práctica de la citología en los últimos tres años. No se excluyeron las mujeres con antecedentes de histerectomía, puesto que ésta información solamente se recogió para las mujeres entre 25 y 49 años; en éste grupo de edad, 953 (2,7 \%) mujeres se habían sometidos a una histerectomía previa. 
Dado que algunas preguntas solo se formularon en las mujeres menores de 49 años, algunos de los resultados se presentan de manera específica para el subgrupo de mujeres entre 25 y 49 años, que ascienden a 23944 mujeres.

\section{Conocimiento y cobertura}

$98,8 \%$ de las mujeres entre 25 y 69 años de edad conoce lo que es la citología y $76,5 \%$ se la han practicado en los últimos tres años. La cobertura en el último año es 50,6 \%. Si a la cobertura reciente se suma la cobertura de hace más de tres años, cerca de $90 \%$ de las mujeres colombianas entre 25 y 69 años se han hecho al menos una citología en la vida.

Según departamentos, la cobertura en los últimos tres años estuvo entre 6,5 \% y 83,7 \% (Tabla 1); solamente Bogotá, Caldas, Risaralda y Quindío tuvieron coberturas superiores a $80 \%$. Algunos departamentos de la Costa Atlántica como Atlántico, Bolívar, La Guajira y Magdalena se destacaron por tener los más bajos porcentajes de cobertura.

La cobertura de citología hace más de tres años varió entre 8,9 y 18,6 \%. El porcentaje de mujeres que nunca se han tomado una citología fue menor en San Andrés y Providencia (4,8 \%) y mayor en La Guajira (19,8 \%).

Factores socio-demográficos relacionados con la práctica reciente de citología

Cuando se exploró la relación entre la cobertura de la citología y algunos factores socio-demográficos se observó una menor cobertura en las mujeres de 55 a 69 años de edad (67,6 \%) y una relación directamente proporcional con el nivel educativo y la riqueza. El tipo de afiliación y el área de residencia también mostraron una asociación con la cobertura en los últimos tres años, con una mejor cobertura en las mujeres afiliadas al Sistema general de seguridad Social en Salud -SGSSS- en cualquiera de los regímenes en contraste con las no afiliadas y las mujeres que residen en áreas rurales; en estos dos grupos de mujeres los porcentajes de nunca haberse hecho una citología alcanzaron las cifras más altas siendo de 17,0 y de 16,1 respectivamente, junto con las que nunca han tenido hijos (21,8 \%) (Tabla 2).

La relación entre el quintil de riqueza y el régimen de afiliación mostró que $60,3 \%$ de las mujeres muy pobres pertenecen al régimen subsidiado y 34,7 \% no están afiliadas; de las mujeres pobres, 47,8 \% están afiliadas al régimen subsidiado y 34,3 \% no están afiliadas. 
Tabla 1. Práctica de la citología del cuello uterino según departamento de residencia en mujeres de 25 a 69 años, Colombia, 2005

\begin{tabular}{|c|c|c|c|c|c|c|}
\hline $\begin{array}{l}\text { Departamento de } \\
\text { residencia }\end{array}$ & $\begin{array}{l}\text { CCU en } \\
\text { los últimos } \\
\text { tres años } \\
(\%)\end{array}$ & $\begin{array}{c}\text { CCU en } \\
\text { el } \\
\text { último } \\
\text { año (\%) }\end{array}$ & $\begin{array}{l}\text { CCU hace } \\
\text { más de } \\
\text { tres años } \\
(\%)\end{array}$ & $\begin{array}{l}\text { Nunca se } \\
\text { ha tomado } \\
\text { la CCU } \\
(\%)\end{array}$ & $\begin{array}{l}\text { Sabe } \\
\text { qué es } \\
\text { la CCU } \\
(\%)\end{array}$ & $\begin{array}{l}\text { Total } \\
\text { mujeres } \\
\text { (n) }\end{array}$ \\
\hline Antioquia & 78,6 & 49,9 & 15,8 & 5,6 & 99,4 & 4448 \\
\hline Atlántico & 66,7 & 42,4 & 18,7 & 14,6 & 99,4 & 1783 \\
\hline Bogotá & 82,7 & 50,3 & 11,2 & 6,1 & 99,6 & 5974 \\
\hline Bolivar & 67,8 & 44,6 & 16,9 & 15,3 & 99,3 & 1611 \\
\hline Boyacá & 70,7 & 43,3 & 11,9 & 17,4 & 94,7 & 1045 \\
\hline $\begin{array}{l}\text { Orinoquia y Ama- } \\
\text { zonia }\end{array}$ & 79,2 & 56,1 & 10,7 & 10,0 & 99,5 & 385 \\
\hline Caldas & 81,3 & 56,8 & 11,4 & 7,3 & 99,9 & 973 \\
\hline Caquetá & 69,5 & 44,4 & 15,7 & 14,8 & 97,0 & 313 \\
\hline Cauca & 68,4 & 48,7 & 12,6 & 19,0 & 95,9 & 915 \\
\hline Cesar & 76,0 & 44,4 & 11,6 & 12,4 & 98,9 & 674 \\
\hline Córdoba & 75,0 & 51,5 & 13,5 & 11,5 & 99,3 & 958 \\
\hline Cundinamarca & 77,7 & 51,7 & 11,4 & 10,9 & 98,9 & 1802 \\
\hline Chocó & 74,3 & 51,8 & 13,1 & 12,7 & 98,3 & 274 \\
\hline Huila & 79,0 & 59,2 & 10,7 & 10,3 & 98,9 & 766 \\
\hline La Guajira & 66,5 & 47,8 & 13,7 & 19,8 & 92,2 & 337 \\
\hline Magdalena & 66,6 & 47,6 & 16,4 & 17,0 & 98,4 & 927 \\
\hline Meta & 79,0 & 53,8 & 10,8 & 10,2 & 97,8 & 566 \\
\hline Nariño & 75,8 & 50,3 & 9,1 & 15,1 & 96,5 & 1353 \\
\hline $\begin{array}{l}\text { Norte de } \\
\text { Santander }\end{array}$ & 67,5 & 46,1 & 15,9 & 16,6 & 98,1 & 1051 \\
\hline Quindío & 83,7 & 63,5 & 9,6 & 6,7 & 99,4 & 529 \\
\hline Risaralda & 81,8 & 64,0 & 11,9 & 6,3 & 99,8 & 853 \\
\hline Santander & 78,3 & 53,7 & 13,8 & 7,8 & 98,7 & 1588 \\
\hline Sucre & 69,1 & 50,9 & 15,5 & 15,4 & 99,5 & 629 \\
\hline Tolima & 72,0 & 44,3 & 15,1 & 12,9 & 97,4 & 1044 \\
\hline Valle & 80,0 & 55,1 & 13,2 & 6,8 & 99,4 & 3774 \\
\hline $\begin{array}{l}\text { San Andrés y } \\
\text { Providencia }\end{array}$ & 78,2 & 53,3 & 17,0 & 4,8 & 100,0 & 75 \\
\hline Colombia & 76,5 & 50,6 & 13,4 & 10,1 & 98,8 & 34.647 \\
\hline
\end{tabular}

Adicionalmente, al hacer la validación del primer modelo de regresión, mediante la evaluación de las correlaciones entre variables independientes, se encontró una alta correlación entre el índice de riqueza y el área de residencia; esta situación obligó a excluir del modelo el área de residencia, puesto que se lograron mejores predicciones con el índice de riqueza que con esta variable.

Los resultados del análisis de regresión mostraron asociaciones importantes entre prácticamente cada una de las variables incluidas y la toma de citología. 
La única variable que no mostró una clara asociación con la práctica de citología fue el estado de salud reportado por la encuestada. Las condiciones que se encontraron asociadas con la ausencia de citología en los últimos tres años fueron: ausencia de afiliación o afiliación al régimen subsidiado, no haber tenido hijos vivos y no haber tenido una consulta de salud en el último año. Las condiciones que se encontraron asociadas con una toma de citología reciente (últimos tres años) fueron: el nivel de riqueza, tener entre 35 y 44 años, tener un nivel educativo universitario o superior y estar en embarazo (Tabla 3).

Tabla 2. Factores socio-demográficos asociados con la práctica de la citología en mujeres de 25 a 69 años, Colombia, 2005

\begin{tabular}{|c|c|c|c|c|c|c|c|}
\hline \multirow[t]{2}{*}{ Variable } & \multirow[t]{2}{*}{$\begin{array}{c}\text { Categorías de la } \\
\text { variable }\end{array}$} & \multicolumn{2}{|c|}{$\begin{array}{l}\text { CCU en los } \\
\text { últimos tres } \\
\text { años }\end{array}$} & \multicolumn{2}{|c|}{$\begin{array}{l}\text { CCU hace más } \\
\text { de tres años }\end{array}$} & \multicolumn{2}{|c|}{$\begin{array}{c}\text { Nunca se ha } \\
\text { tomado la } \\
\text { CCU }\end{array}$} \\
\hline & & $\mathrm{N}$ & $\%$ & $\mathrm{n}$ & $\%$ & $\mathrm{n}$ & $\%$ \\
\hline \multirow{4}{*}{$\begin{array}{l}\text { Grupos de } \\
\text { edad }\end{array}$} & De 25 a 34 años & 8007 & 76,7 & 1002 & 9,6 & 1435 & 13,7 \\
\hline & De 35 a 44 años & 7864 & 80,5 & 1250 & 12,8 & 651 & 6,7 \\
\hline & De 45 a 54 años & 6312 & 78,5 & 1156 & 14,4 & 569 & 7,1 \\
\hline & De 55 a 69 años & 4324 & 67,6 & 1232 & 19,2 & 845 & 13,2 \\
\hline \multirow{5}{*}{$\begin{array}{l}\text { Afiliación al } \\
\text { SGSSS }\end{array}$} & Contributivo & 12387 & 85,9 & 1404 & 9,7 & 637 & 4,4 \\
\hline & Subsidiado & 7594 & 73,7 & 1390 & 13,5 & 1326 & 12,9 \\
\hline & Especial & 980 & 87,8 & 105 & 9,4 & 31 & 2,8 \\
\hline & No está afiliada & 5475 & 63,0 & 1728 & 19,9 & 1486 & 17,1 \\
\hline & No sabe & 71 & 68,4 & 12 & 11,3 & 21 & 20,3 \\
\hline \multirow{3}{*}{$\begin{array}{l}\text { Área de } \\
\text { residencia }\end{array}$} & Urbana & 21107 & 78,0 & 3662 & 13,5 & 2276 & 8,4 \\
\hline & Rural & 5400 & 71,0 & 977 & 12,9 & 1224 & 16,1 \\
\hline & Muy pobre & 3215 & 64,5 & 718 & 14,4 & 1049 & 21,1 \\
\hline \multirow{4}{*}{$\begin{array}{l}\text { Quintil de } \\
\text { índice de } \\
\text { pobreza }\end{array}$} & Pobre & 4428 & 70,9 & 937 & 15,0 & 884 & 14,1 \\
\hline & Medio & 5343 & 75,4 & 1064 & 15,0 & 680 & 9,6 \\
\hline & Rico & 6226 & 79,6 & 1059 & 13,5 & 540 & 6,9 \\
\hline & Muy rico & 7295 & 85,8 & 861 & 10,1 & 348 & 4,1 \\
\hline \multirow{3}{*}{$\begin{array}{l}\text { consulta de } \\
\text { salud en el } \\
\text { último año }\end{array}$} & $\mathrm{Si}$ & 21754 & 82,5 & 2724 & 10,3 & 1906 & 7,2 \\
\hline & No & 4691 & 57,4 & 1906 & 23,3 & 1582 & 19,3 \\
\hline & No sabe & 62 & 73,3 & 10 & 11,6 & 13 & 15,1 \\
\hline \multirow{5}{*}{$\begin{array}{l}\text { Estado de } \\
\text { salud }\end{array}$} & Excelente & 1133 & 79,1 & 178 & 12,5 & 121 & 8,4 \\
\hline & Muy bueno & 2156 & 79,7 & 326 & 12,1 & 223 & 8,2 \\
\hline & Bueno & 15289 & 77,3 & 2491 & 12,6 & 1988 & 10,1 \\
\hline & Regular & 7275 & 74,1 & 1496 & 15,2 & 1044 & 10,6 \\
\hline & Malo & 654 & 70,5 & 149 & 16,0 & 126 & 13,5 \\
\hline \multirow{4}{*}{$\begin{array}{l}\text { Nivel } \\
\text { educativo * }\end{array}$} & $\begin{array}{l}\text { Primaria o menos } \\
\text { Secundaria o }\end{array}$ & 5828 & 74,9 & 1055 & 13,6 & 898 & 11,5 \\
\hline & tecnológica & 9851 & 80,3 & 1369 & 11,2 & 1040 & 8,5 \\
\hline & superior & 2420 & 85,4 & 197 & 70 & 215 & 76 \\
\hline & Sin información & 425 & 58,6 & 114 & 15,7 & 186 & 25,6 \\
\hline \multirow{2}{*}{$\begin{array}{l}\text { Ha tenido hijos } \\
\text { vivos * }\end{array}$} & Sí & 16625 & 79,3 & 2571 & 12,3 & 1764 & 8,4 \\
\hline & No & 1898 & 72,0 & 164 & 6,2 & 575 & 21,8 \\
\hline \multirow{3}{*}{$\begin{array}{l}\text { Embarazo } \\
\text { actual * }\end{array}$} & Sí & 581 & 76,1 & 68 & 8,9 & 114 & 14,9 \\
\hline & No & 17871 & 78,6 & 2653 & 11,7 & 2210 & 9,7 \\
\hline & No sabe & 72 & 71,4 & 13 & 12,9 & 16 & 15,7 \\
\hline
\end{tabular}


En relación con el pago que las mujeres debieron hacer por la citología, 78,6 \% reportaron que no pagaron nada, 3,7 \% tuvieron que hacer algún pago parcial y $17,7 \%$ la pagaron en su totalidad. Este porcentaje fue significativamente mayor entre las mujeres no afiliadas y del régimen especial en el cual 33,1 \% y 21,1 \% respectivamente pagaron la totalidad de la citología. En las mujeres del régimen contributivo, 13,6 \% pagaron la citología; este porcentaje fue de 12,6\% en mujeres del régimen subsidiado.

Tabla 3. Práctica de la citología en los tres años anteriores en mujeres de 25 a 49 años según variables socio-demográficas

\begin{tabular}{|c|c|c|c|c|c|c|}
\hline \multirow[b]{2}{*}{ Variable } & \multirow[b]{2}{*}{ Categoría } & \multicolumn{3}{|c|}{ CCU últimos tres años } & \multirow[b]{2}{*}{ O.R. } & \multirow[b]{2}{*}{$P$} \\
\hline & & $\begin{array}{c}\text { Si } \\
(\%)\end{array}$ & $\begin{array}{l}\text { No } \\
(\%)\end{array}$ & & & \\
\hline \multirow{3}{*}{$\begin{array}{l}\text { Grupos de } \\
\text { edad }\end{array}$} & De 25 a 34 años & 76,6 & 23,4 & 10395 & 1 & 0,00 \\
\hline & De 35 a 44 años & 80,4 & 19,6 & 9406 & 0,83 & 0,00 \\
\hline & De 45 a 54 años & 78,9 & 21,1 & 3797 & 0,97 & 0,51 \\
\hline \multirow{4}{*}{$\begin{array}{l}\text { Nivel } \\
\text { educativo }\end{array}$} & Primaria o menos & 74,9 & 25,1 & 7781 & 1 & 0,00 \\
\hline & Secundaria o tecnológica & 80,3 & 19,7 & 12260 & 0,94 & 0,16 \\
\hline & Universitaria o superior & 85,4 & 14,6 & 2832 & 0,83 & 0,02 \\
\hline & formación & 58,6 & 41,4 & 724 & 1,69 & 0,00 \\
\hline \multirow{5}{*}{$\begin{array}{l}\text { Afiliación } \\
\text { al SGSSS }\end{array}$} & Contributivo & 87,4 & 12,6 & 9406 & 1 & 0,00 \\
\hline & Subsidiado & 76,6 & 23,4 & 7032 & 1,58 & 0,00 \\
\hline & Especial & 89,8 & 10,2 & 662 & 0,90 & 0,42 \\
\hline & No está afiliada & 66,3 & 33,7 & 6420 & 2,18 & 0,00 \\
\hline & No sabe & 76,5 & 23,5 & 78 & 1,33 & 0,31 \\
\hline \multirow{5}{*}{$\begin{array}{l}\text { Quintil de } \\
\text { indice de } \\
\text { riqueza }\end{array}$} & Muy pobre & 68,2 & 31,8 & 3371 & 1 & 0,00 \\
\hline & Pobre & 74,2 & 25,8 & 4478 & 0,81 & 0,00 \\
\hline & Medio & 77,4 & 22,6 & 4984 & 0,76 & 0,00 \\
\hline & Rico & 81,1 & 18,9 & 5325 & 0,68 & 0,00 \\
\hline & Muy r & 86,9 & 13,1 & 5439 & 0,55 & 0,00 \\
\hline \multirow{2}{*}{$\begin{array}{l}\text { Ha tenido } \\
\text { hijos vivos }\end{array}$} & $\mathrm{Si}$ & 79,3 & 20,7 & 20960 & 1 & 0,00 \\
\hline & No & 72,0 & 28,0 & 2638 & 2,05 & 0,00 \\
\hline \multirow{3}{*}{$\begin{array}{l}\text { Embarazo } \\
\text { actual }\end{array}$} & $\mathrm{Si}$ & 76,1 & 23,9 & 763 & 1 & 0,00 \\
\hline & No & 78,6 & 21,4 & 22734 & 0,76 & 0,00 \\
\hline & No sabe & 71,4 & 28,6 & 101 & 1,09 & 0,72 \\
\hline \multirow{3}{*}{$\begin{array}{l}\text { Consulta } \\
\text { de salud } \\
\text { último año }\end{array}$} & Si & 84,9 & 15,1 & 17457 & 1 & 0,00 \\
\hline & No & 60,0 & 40,0 & 6074 & 3,14 & 0,00 \\
\hline & No sabe & 74,8 & 25,2 & 67 & 1,80 & 0,04 \\
\hline \multirow{3}{*}{$\begin{array}{l}\text { Estado de } \\
\text { salud }\end{array}$} & Excelente o muy bueno & 80,0 & 20,0 & 3151 & 1 & 0,77 \\
\hline & Bueno & 78,4 & 21,6 & 14519 & 1,02 & 0,68 \\
\hline & Regular o malo & 77,9 & 22,1 & 5928 & 1,00 & 0,94 \\
\hline
\end{tabular}

Factores de riesgo para cáncer de cuello uterino

La encuesta permitió evaluar la prevalencia de cuatro factores de riesgo establecidos para cáncer de cuello uterino $(14,17)$, a saber: inicio de relaciones sexuales antes de los 15 años, haber tenido más de tres hijos nacidos vivos, ausencia de citología en los últimos tres años y pobreza. Los dos factores de 
riesgo con mayor prevalencia en prácticamente todos los departamentos fueron la multiparidad y la pobreza.

Al evaluar la presencia de dos o más factores de riesgo simultáneos para cáncer de cuello uterino se encontró una prevalencia de 31 \% para las mujeres entre 25 y 49 años; este porcentaje fue significativamente menor para Bogotá, Quindío y Valle; en contraste Chocó, Caquetá, Cauca y Cesar muestran condiciones de riesgo más altas con porcentajes entre 60 \% y 50 \% (Tabla 4).

Tabla 4. Prevalencia (\%) de factores de riesgo para cáncer de cuello uterino en mujeres entre 25 y 69 años según departamento de residencia Colombia, 2005.

\begin{tabular}{|c|c|c|c|c|c|}
\hline $\begin{array}{l}\text { Departamento de } \\
\text { residencia }\end{array}$ & $\begin{array}{l}\text { IRS antes } \\
\text { de los } 15 \\
\text { años * }\end{array}$ & $\begin{array}{l}\text { Más de } \\
\text { tres hijos } \\
\text { nacidos } \\
\text { vivos * }^{*}\end{array}$ & $\begin{array}{c}\text { Nivel de } \\
\text { riqueza: } \\
\text { pobre y } \\
\text { muy } \\
\text { pobre }\end{array}$ & $\begin{array}{l}\text { Ausencia } \\
\text { de CCU } \\
\text { en los } \\
\text { últimos } \\
\text { tres años }\end{array}$ & $\begin{array}{l}\text { Dos o más } \\
\text { factores de } \\
\text { riesgo } \\
\text { simultáneos* }\end{array}$ \\
\hline Antioquia & 10,1 & 39,6 & 29,2 & 21,4 & 28,6 \\
\hline Atlántico & 8,3 & 43,2 & 30,8 & 33,3 & 33,0 \\
\hline Bogotá & 8,3 & 35,0 & 6,9 & 17,3 & 14,5 \\
\hline Bolívar & 11,0 & 47,4 & 49,3 & 32,2 & 40,2 \\
\hline Boyacá & 9,1 & 52,9 & 58,4 & 29,3 & 45,4 \\
\hline Orinoquia y Amazonia & 17,8 & 42,6 & 29,1 & 20,8 & 29,6 \\
\hline Caldas & 10,1 & 38,6 & 32,5 & 18,7 & 26,6 \\
\hline Caquetá & 18,9 & 62,0 & 57,6 & 30,5 & 55,6 \\
\hline Cauca & 17,7 & 52,4 & 56,2 & 31,6 & 50,9 \\
\hline Cesar & 17,7 & 56,6 & 56,9 & 24,0 & 50,1 \\
\hline Córdoba & 14,3 & 50,4 & 68,2 & 25,0 & 51,6 \\
\hline Cundinamarca & 12,2 & 49,2 & 36,3 & 22,3 & 35,1 \\
\hline Chocó & 21,8 & 56.4 & 76.5 & 25,7 & 61,0 \\
\hline Huila & 10,7 & 52,0 & 44,3 & 21,0 & 40,7 \\
\hline La Guajira & 12,7 & 51,8 & 48,6 & 33,5 & 43,2 \\
\hline Magdalena & 14,3 & 54,5 & 53.9 & 33,4 & 48.9 \\
\hline Meta & 19,0 & 48,4 & 32,4 & 21,0 & 34,1 \\
\hline Nariño & 12,6 & 40,3 & 50,6 & 24,2 & 36,2 \\
\hline Norte de Santander & 10,2 & 49,8 & 34,6 & 32,5 & 38,5 \\
\hline Quindio & 12,2 & 30,4 & 14,8 & 16,3 & 17,6 \\
\hline Risaralda & 13,1 & 33,1 & 27,5 & 18,2 & 23,4 \\
\hline Santander & 8,6 & 43,5 & 32,6 & 21,7 & 30,9 \\
\hline Sucre & 9,9 & 49,9 & 52,9 & 30,9 & 44,9 \\
\hline Tolima & 17,9 & 44,6 & 37,3 & 28,0 & 37,2 \\
\hline Valle & 13,2 & 33,9 & 19,6 & 20,0 & 22,8 \\
\hline \multicolumn{6}{|l|}{ San Andrés y } \\
\hline Providencia & 14,0 & 35,3 & 27,4 & 21,8 & 27,4 \\
\hline Colombia & 11,6 & 42,4 & 32,4 & 23,5 & 31,0 \\
\hline
\end{tabular}

* Estas variables aplican solo a mujeres entre 25 y 49 años 


\section{DISCUSIÓN}

Los resultados revelan que prácticamente todas las mujeres colombianas entre 25 y 69 años conocen la citología y la variabilidad es mínima entre departamentos; estos resultados son similares a los disponibles anteriormente en la Encuesta Nacional de Factores de Riesgo (ENFREC II), realizada en 1998, en la cual el conocimiento sobre la citología fue 96,1 \% en mujeres entre 18 y 69 años (15). Estos resultados contrastan con algunos estudios realizados en otros países de Latinoamérica como Brasil, en los que $14 \%$ de las mujeres no tenían conocimiento sobre la citología (18).

La cobertura de citología en los últimos tres años para Colombia obtenida en este estudio es de 76,3 \% lo que también muestra un incremento en relación con la cobertura reportada en ENFREC II que fue de 68,4 \% (15). Si se compara con información disponible de otros países, también es satisfactoria: la cobertura en los últimos tres años en Estados Unidos es 83 \% (19) y en Sao Paulo, Brasil, es de 77,3 \% (20).

Al examinar las diferencias Departamentales en la cobertura, las mayores prevalencias en Bogotá y los Departamentos de Caldas, Quindío y Risaralda podrían reflejar el resultado de una mayor conciencia del problema del cáncer de cuello uterino y de acciones coordinadas para tal fin. En el caso de Bogotá existen normas específicas para la detección temprana y hay un esfuerzo de acciones coordinadas para el control del cáncer de cuello uterino (21) mientras que en el eje cafetero hay evidencia sobre la existencia de grupos reconocidos en investigación (22).

Aunque en algunos Departamentos como Cauca, La Guajira y Magdalena sí se observó una relación directa entre el porcentaje de mujeres que nunca se han tomado una citología y las tasas altas mortalidad por cáncer de cuello uterino disponibles para el quinquenio 1995-1999 $(23,24)$ ésta relación no fue contundente ni general, lo que puede explicarse por múltiples factores entre los cuales están la calidad de la certificación de la muerte, el acceso al diagnóstico definitivo y el acceso al tratamiento oportuno una vez establecido el diagnóstico.

A pesar de las restricciones impuestas por la normatividad al no haber incluido a la población no afiliada (10), los resultados del presente análisis muestran porcentajes altos de cobertura en los últimos tres años independientemente de la afiliación o no al SGSSS, aunque sí es menor en la

población no asegurada. Resulta preocupante que el 33 \% de las mujeres no 
afiliadas paguen la toma de la citología en su totalidad; esto evidencia la limitación al haberlas excluido de la norma pero además sugiere que el mecanismo de financiación con subsidio a la demanda previsto a cargo de los departamentos para esta actividad en las mujeres no afiliadas (25), no funciona debidamente. Resultados preliminares de otro estudio en un municipio colombiano respaldan esta hipótesis, ya que se encontró que las instituciones prestadoras de servicios de toma de citología no aplican el mecanismo previsto y aluden a la insuficiencia de los recursos situados (C. Wiesner, comunicación personal, Instituto nacional de Cancerología, julio 2006).

Con respecto a los factores asociados con la toma de citología, la menor cobertura observada en mujeres entre 50 y 69 años de edad es un fenómeno que se ha observado en muchos programas de tamización que muestran que la cobertura disminuye con la edad (26), pero es preocupante debido a que las tasas de incidencia de cáncer de cuello uterino específicas por edad, muestran para Colombia un patrón de incremento en las mujeres mayores de 55 años (5). De otro lado, la menor cobertura en mujeres de mayor edad debe interpretarse con cautela puesto que la encuesta no indagó sobre la histerectomía en las mujeres mayores de 50 años; si la cantidad de mujeres con histerectomía previa constituye un porcentaje alto, puede llevar a subestimar la cobertura en este grupo, aspecto que se detalla con mayor profundidad en las limitaciones.

La posible asociación entre nivel educativo y toma de citología podría estar explicada por una tercera variable, el nivel de riqueza. Sin embargo, esta hipótesis no resultó cierta para el tipo de afiliación; después de controlar por el nivel de riqueza y el nivel educativo, aún se encontró una asociación importante entre la falta de afiliación y la afiliación al régimen subsidiado con la ausencia de citología en los últimos tres años. Esta información implica que hay barreras para el acceso a los servicios de tamización para cáncer de cuello uterino en este tipo de población, que es precisamente el grupo poblacional con mayor riesgo para este cáncer. Información no publicada, proveniente del análisis de las bases de datos de defunciones del país, muestra cómo del total de muertes por cáncer de cuello uterino en los años 2000-2003, 42,8 \% eran mujeres afiliadas al régimen subsidiado, 20,2 \% no estaban aseguradas y 26,8 \% eran del régimen contributivo.

El hecho de haber asistido a una consulta de salud en el último año mostró estar asociado con la toma de citología; ello se puede atribuir a un proceso efectivo de inducción de la demanda de citología en los servicios de salud, más que a otras condiciones tales como el nivel educativo o las condiciones 
económicas de las mujeres que asistieron a consultas de salud en el último año. La relación de una mayor cobertura con el antecedente de consulta de salud se ha observado también en otros estudios a nivel nacional y latinoamericano $(27,28)$.

\section{Limitaciones}

La primera limitación importante de mencionar es la posible sobreestimación de las cifras de cobertura, reportado en otros estudios por las dificultades en recordar la fecha de la última citología y el deseo de la encuestada de responder de una manera socialmente aceptable (29).

En el presente estudio, la exploración de asociaciones entre variables posiblemente relacionadas con la cobertura de citología en los últimos tres años y la descripción de factores de riesgo se hicieron solamente para mujeres entre 25 y 49 años (que tenían la información completa para todas las variables), por lo que los hallazgos son ciertos para este grupo, pero no reflejan el comportamiento de las mujeres de 50 o más años. Esta limitación debe considerarse en la planeación de la siguiente encuesta de demografía y salud, así como también debería incluirse el número de compañeros sexuales en la vida, que es un factor de riesgo muy importante para cáncer de cuello uterino.

De otro lado hay limitaciones para la interpretación de las coberturas en las regiones de Orinoquia y Amazonia, considerando que en estas regiones más del 50 \% de la población quedó excluida al descartar a la población rural del universo de estudio.

Una última limitación del estudio se refiere a la ausencia de información sobre histerectomía en el grupo de mujeres mayores de 50 años que conlleva una posible subestimación de la cobertura de citologías. Las mujeres que han sido sometidas a histerectomía no son objeto del programa de tamización (13) y es particularmente en el grupo de mayores de 50 donde el porcentaje puede estar entre 30 y $40 \%$ (19).

Finalmente, no se pudo analizar la regularidad de la toma, debido a que las categorías de respuesta ("más de una vez al año", "una vez al año", "rara vez") estaban sujetas a una gran variabilidad y diversidad de conceptos. La regularidad en la toma es importante si se considera la evidencia disponible del efecto protector aún con una citología cada diez años después de una citología negativa 
Agradecimientos. A Gabriel Ojeda, Myriam Ordoñez y Rocío Murad de Profamilia, Bogotá, por permitir al INC comentarios sobre los formularios de recolección, facilitar la base de datos y explicación en relación con los factores de expansión y la estructura de la base de datos de la encuesta ENDS.

\section{REFERENCIAS}

1. IARC Scientific Publication Cervical Cancer Screening Handbooks on prevention

2. Anderson GH, Boyes D, Benedit JL. Organization and results of the cervical citology screening programme in British Columbia. BMJ 1988;296:975-978.

3. Duguid H, Duncan I, Currie J. Screening for cervical cancer intraepithelial neoplasia in Dundee and Augus 1962-1981 and its relation with cervical invasive cancer. Lancet 1985;2:1053-1058.

4. Murillo RH. La prevención del cáncer: más allá de las pruebas de tamizaje. Rev. Colomb Cancerol 2003;7(2):23-33.

5. Globocan 2002 [programa de computador]. Lyon: International Agency for Research on Cancer; 2004.

6. El cáncer en cifras [Internet]. Disponible en: http//cancer.gov.co. Consultado Julio 2006.

7. Piñeros M, Ferlay J, Murillo R. Incidencia estimada y mortalidad por cáncer en Colombia: 1995-1999. Bogotá: Imprenta Nacional; 2005.

8. Piñeros M, Hernández G Bray FI. Increasing mortality rates of common malignancies in Colombia: an emerging problem. Cancer 2004;101(10):2285-92.

9. República de Colombia, Sistema Nacional de Salud, Instituto Nacional de Cancerología. Normas técnicas y administrativas. Guía de Implantación. Detección y Control de cáncer de cuello uterino. Bogotá: Imprenta Nacional; 1994.

10. República de Colombia, Ministerio de Salud. Normas técnicas y guías de atención. Tomo I. Serie Documentos Técnicos. Bogotá: Trazo Digital Impresores; 2000.

11. República de Colombia, Ministerio de Salud. Política de Salud Sexual y Reproductiva. Bogotá: Ministerio de Salud; 2000.

12. República de Colombia Ministerio de Salud. Resolución 3384 de 2000 por la cual se modifican parcialmente las resoluciones 412 y 1745 de 2000 y se deroga la resolución 1078 de 2000. Diario Oficial 44276, diciembre de 2000. Bogotá: Imprenta Nacional; 2000.

13. Screening for cervical cancer [Internet]. Disponible en: http://www.arqh.gov/clinic/ uspstf/uspcerv.htm. Consultado en agosto 2006.

14. Colditz GA, Atwood KA, Emmons K, Monson RR, Willett WC, Trichopoulos D, et al. Harvard report on cancer prevention volume 4: Harvard Cancer Risk Index. Risk Index Working Group, Harvard Center for Cancer Prevention.Cancer Causes Control. 2000 Jul;11(6):477-88. 
15. Ministerio de Salud, Centro Nacional de Consultoría. Conocimientos, actitudes y prácticas en detección temprana de cáncer cérvico-uterino y de mama. II Estudio Nacional de Factores de Riesgo de Enfermedades Crónicas (ENFREC II). Tomo IV. Bogotá D. C.: Ministerio de Salud; 1999.

16. Ojeda G, Ordóñez M, Ochoa L, Samper B, Sánchez F. Salud sexual y reproductiva: Resultados Encuesta Nacional de Demografía y Salud 2005. Asociación Probienestar de la Familia Colombiana (Profamilia). Profamilia, Bienestar Familiar, United State Agency for Internacional Development, Ministerio de la Protección Social; 2005.

17. Castellsagué $X$, Bosch $X$, Munoz N. Environmental co-factors in HPV carcinogenesis. Virus Res 2002;89(2):191-9.

18. De Quadros CA, Victora CG, da Costa JS. Coverage and focus of a cervical cancer prevention program in southern Brazil. Rev Panam Salud Publica 2004;16(4):223-32.

19. Hewitt M, Devesa SS, Breen N. Cervical cancer screening among U.S. women: analyses of the 2000 National Health Interview Survey. Prev Med. 2004;39(2):270-8.

20. Pinho AA, Franca JI, Schraiber LB, D'Oliveira AF. Coverage and factors involved in submitting to the Papanicolaou test in the Municipality of Sao Paulo. Cad Saude Publica 2003;19 Suppl 2:S303-13.

21. Alcaldía Mayor de Bogotá, Secretaría de Salud. Manual de normas técnicoadministrativas para el programa de detección y control del cáncer de cuello uterino. Bogotá; 2005.

22. Murillo R, Gamboa O. Investigación y situación del cáncer en Colombia. Innovación y Ciencia 2006;XVII(1):39-47.

23. Murillo R, Piñeros M. Hernández G Atlas de Mortalidad en Colombia: Bogotá: Instituto Nacional de Cancerología, Instituto Geográfico Agustín Codazzi; 2003. 